\title{
Does investment in the health sector promote or inhibit economic growth?
}

\author{
Aaron Reeves ${ }^{*}$, Sanjay Basu ${ }^{2,3}$, Martin McKee ${ }^{3}$, Christopher Meissner ${ }^{4}$ and David Stuckler ${ }^{1,3}$
}

\begin{abstract}
Background: Is existing provision of health services in Europe affordable during the recession or could cuts damage economic growth? This debate centres on whether government spending has positive or negative effects on economic growth. In this study, we evaluate the economic effects of alternative types of government spending by estimating "fiscal multipliers" (the return on investment for each $\$ 1$ dollar of government spending).

Methods: Using cross-national fixed effects models covering 25 EU countries from 1995 to 2010, we quantified fiscal multipliers both before and during the recession that began in 2008.

Results: We found that the multiplier for total government spending was 1.61 (95\% Cl: 1.37 to 1.86), but there was marked heterogeneity across types of spending. The fiscal multipliers ranged from -9.8 for defence (95\% Cl: -16.7 to -3.0 ) to 4.3 for health ( $95 \% \mathrm{Cl}: 2.5$ to 6.1). These differences appear to be explained by varying degrees of absorption of government spending into the domestic economy. Defence was linked to significantly greater trade deficits $(\beta=-7.58$, $p=0.017$ ), whereas health and education had no effect on trade deficits ( $p_{\text {education }}=0.62 ; p_{\text {health }}=0.33$ ).

Conclusion: Our findings indicate that government spending on health may have short-term effects that make recovery more likely.
\end{abstract}

Keywords: Health spending, Government spending, Economic growth

\section{Background}

The Great Recession that beset Europe and North America since 2007 has sparked widespread debate about alternative approaches to achieving economic recovery. Much of the discussion has centred on the question of whether government spending will promote [1-3] or inhibit economic growth [4,5]. Critics of government spending argue, first, that such spending has an immediate effect of increasing debt which, if associated with a loss of confidence by investors, will also increase the cost of servicing that debt as a consequence of increased interest rates [6,7]. Second, they argue that, by "crowding out" private markets and, by implication, their assumed greater efficiency, they will inhibit the growth necessary for recovery [8]. In contrast, advocates of greater spending during

\footnotetext{
* Correspondence: aaron.reeves@sociology.ox.ac.uk

'Department of Sociology, University of Oxford, Manor Road Building, Manor Road, Oxford OX1 3UQ, England

Full list of author information is available at the end of the article
}

recessionary periods, which are characterized by unemployment and deficits, argue that the effects of short term increases in borrowing will be compensated for by higher growth resulting from a high marginal propensity to consume, generating positive cycles of consumption and employment growth [9]. Many of these arguments have been tested empirically. Contrary to what is claimed by the advocates of austerity, increased debt is not consistently associated with high interest rates nor lower economic growth rates [10-12]. Similarly, rather than crowding out private markets, government spending, at least on physical infrastructure, seems to increase the productivity of private capital $[13,14]$. However, other aspects of stimulus spending have been subject to less attention from researchers.

One aspect that has received less attention is the potential return on government investment in the health sector. The fiscal multiplier is an estimate of the effect of government spending on economic growth. A multiplier greater than 1 corresponds to a positive growth stimulus
C Biomed Central

(C) 2013 Reeves et al.; licensee BioMed Central Ltd. This is an Open Access article distributed under the terms of the Creative Commons Attribution License (http://creativecommons.org/licenses/by/2.0), which permits unrestricted use, distribution, and reproduction in any medium, provided the original work is properly cited. 
(returning more than $\$ 1$ for each dollar invested), whereas a multiplier less than one reflects a net loss from spending. Prior studies have estimated the aggregate multipliers from overall government spending [15-19], most notably in a recent study by two senior economists at the International Monetary Fund that suggested that the multiplier was greater than one, higher than the previously assumed multiplier of 0.5-0.6 [20]. It is obvious that some sectors will have greater capacity to translate additional funds into new employment opportunities or increase incomes domestically and thus intuitive that such expenditure will achieve greater fiscal multipliers [16,21]. However, to our knowledge no study has yet to comparatively estimate the growth effects of differing types of government spending.

While this paper cannot hope to resolve the debate between those advocating greater austerity to reduce levels of debt and those who see government spending as a way of encouraging recovery (and in turn the debt), it is possible to examine what any government that wished to adopt the latter course should spend its money on to achieve the greatest return on investment. Health, education, and social welfare sectors are currently experiencing significant cuts in several European countries, either as a result of political decisions by national governments or, in the case of countries subject to bail outs, by the troika of the European Commission, European Central Bank, and International Monetary Fund [22]. There is growing evidence that these cuts are having an adverse effect on the health of populations [23]. The question we ask here is whether they are also damaging the prospects for economic growth.

\section{Methods}

In the first step of the analysis, we used comparative cross-national data on sector-specific funding [24] among 25 EU countries from 1995 to 2010 to estimate empirically the magnitude and direction of fiscal multipliers in health, defence, education, and other key government spending sectors (see Table 1 for definitions of budget categories).

To estimate fiscal multipliers empirically in nonrecession periods, we estimated a weighted-average of country-specific slopes ('within-country' variation). Government spending may correlate with unobserved factors that also independently affect economic growth. To address potential confounding factors, some studies apply mathematical simulation models, relying on theoretical assumptions $[25,26]$. Vector autoregressive models have been applied to quarterly data for small numbers of countries, but for annual data with larger numbers of countries fixed effects models are more consistent. Casestudies of small economies have been applied to correct for potential confounding factors, but these micro studies lack generalisability to larger economies. Instead, to correct for these potential confounding factors, we correct for total
Table 1 Types of government spending

\begin{tabular}{|c|c|}
\hline \multicolumn{2}{|c|}{ Types of government spending } \\
\hline Health & $\begin{array}{l}\text { Government outlays on health include expenditures } \\
\text { on services provided to individual persons and } \\
\text { services provided on a collective basis; Medical } \\
\text { products, appliances, and equipment; Outpatient, } \\
\text { hospital, and public health services }\end{array}$ \\
\hline Education & $\begin{array}{l}\text { Pre-primary, primary, secondary, post-secondary, } \\
\text { non-tertiary, tertiary education Provision of } \\
\text { education not definable by level; Subsidiary services } \\
\text { to education }\end{array}$ \\
\hline Culture & $\begin{array}{l}\text { Recreational, sporting, cultural, broadcasting, } \\
\text { publishing, religious and other community services; } \\
\text { R\&D recreation, culture, and religion }\end{array}$ \\
\hline $\begin{array}{l}\text { Housing and } \\
\text { community }\end{array}$ & $\begin{array}{l}\text { Housing and community development; Water } \\
\text { supply and street lighting }\end{array}$ \\
\hline $\begin{array}{l}\text { General public } \\
\text { services }\end{array}$ & $\begin{array}{l}\text { Executive and legislative organs, financial and fiscal } \\
\text { affairs, external affairs, Public debt transactions; } \\
\text { General and public services; Foreign economic aid } \\
\text { Police, fire, and prison services; Law courts; R\&D } \\
\text { public order and safety }\end{array}$ \\
\hline Defence & $\begin{array}{l}\text { Military and civil defence; foreign aid defence; R\&D } \\
\text { Defence }\end{array}$ \\
\hline Environment & $\begin{array}{l}\text { Waste and waste water management; Pollution } \\
\text { abatement. Protection of biodiversity and landscape }\end{array}$ \\
\hline Social protection & $\begin{array}{l}\text { Sickness and disability, old age, survivors, family and } \\
\text { children, unemployment, housing, and social } \\
\text { exclusion }\end{array}$ \\
\hline Economic affairs & $\begin{array}{l}\text { General economic, commercial, and labour affairs; } \\
\text { agriculture, forestry, fishing, hunting, fuel and } \\
\text { energy, mining, manufacturing, construction, } \\
\text { transport, communication and other industries. }\end{array}$ \\
\hline
\end{tabular}

Source: EuroStat 2013 edition.

government spending as well as between-country heterogeneity by using fixed effects models $[27,28]$, covering the years 1995-2007 as follows:

$$
G D P_{i t}=\alpha+\beta G_{i j t}+\phi\left(\left(\sum_{j} G_{i j t}\right)-G_{i j t}\right)+\mu_{i}+\varepsilon_{i t}
$$

Here $i$ is country, $j$ is type of government spending as described in Table 1, and $t$ is year. GDP is Gross Domestic Product and $G$ is government spending, both in purchasing-power-parity and constant international 2005 dollars. $\beta$ is the estimate fiscal multiplier, where $\beta=1$ is no effect, $\beta>1$ is a multiplicative effect, and $\beta<1$ is a contractionary effect, and $\phi$ is the fiscal multiplier for the total of all other types of government spending. Standard errors were clustered by country to reflect non-independence of sampling.

In the second stage of the analysis, to test whether fiscal multipliers differ in recession and non-recessionary periods, we quantified fiscal multipliers from the recessionary period between 2008 and 2010, then compared these estimated fiscal multipliers with those from prerecession models. We also use out-of sample prediction 
with observed economic outcomes between 2008 and 2010 and for non-EU economies of the U.S. and Japan. In subsequent analyses, to assess whether specific multipliers differed across recessionary and non-recessionary periods, we added an interaction term for the recessionary years. All analyses were performed using STATA v12.1.

\section{Results}

\section{Estimating fiscal multipliers by type of government} spending, pre-recession 1995-2007

The forest plot in Figure 1 displays the estimated fiscal multiplier across eight types of government spending, which range from -9.8 and 8.4 among various government spending sectors. Overall, we estimated the fiscal multiplier for total government spending as 1.61 (95\% CI: 1.37 to 1.86 ), which is consistent with recent independent estimates of the aggregate multiplier [17].

The magnitude of fiscal multipliers differed significantly by type of government spending. As shown in Figure 1, we estimated a negative fiscal multiplier of defence spending (-9.8, 95\% CI: -16.7 to -3.0$)$, while the largest positive fiscal multipliers were in the sectors of health $(4.32,95 \% \mathrm{CI}$ : 2.51 to 6.14 ) and education (8.24, 95\% CI: 3.94 to 12.54 ).

To further evaluate potential mediating factors, we included a standard set of determinants of economic growth, including time dummies, interest rates, unemployment, trade balance, and domestic investment (Figure 2a). All factors had associations in the expected direction (e.g. higher unemployment and interest rates were negative, whereas greater savings, investment, and net exports were positive) (see Tables $2 \mathrm{a}-\mathrm{j}$ ). Consistent with a domestic multiplier mechanism, we found that when we adjusted for unemployment, the health and education multipliers were attenuated, but adjusting for trade balance had no effect. In contrast, when after adjusting for the trade balance, the defence spending multiplier was attenuated $(\beta=-3.62$, Table $2 \mathrm{c})$.

We found evidence that the size of the fiscal multiplier was partly mediated by the degree of domestic absorption of government spending. Tradable components of government spending, like defence spending, were linked to significantly more negative trade balance $(\beta=-7.58$, $\mathrm{p}=0.017$ ) (see Additional file 1: Web Appendix 1). However, non-tradable areas of the budget, including health and education had no significant association with the trade balance $\left(\mathrm{p}_{\text {education }}=0.62\right.$; $\mathrm{p}_{\text {health }}=0.33$ ) (see Additional file 1: Web Appendix 1).

\section{Observed fiscal multiplier for total government spending, 2008-2010}

As a final test, we performed two out-of-sample predictions using our estimates from the 1995-2007 period. First, we quantified the fiscal multiplier for total government spending during the current recessionary period by identifying the period of significant changes in government spending. Panel a of Figure 3 presents a heat map showing patterns of government spending change in the $\mathrm{EU}$ (blue = increased, red = decreased spending). Europe's governments have differed considerably in their patterns of expenditure, both overall and across sectors. Greece, Luxembourg, and Hungary have implemented large reductions, whereas Germany, Finland, Poland, and Sweden implemented significant spending increases. In general, community and general public services (e.g. civil service) have seen the largest budget shifts in both positive and negative directions. Social protection spending has tended to increase during the recession, while defence spending has tended to decline slightly.

Figure 4 presents the association of changes in government spending and in Gross Domestic Product $(\beta=1.05$, 95\% CI: 0.49 to $1.61, \mathrm{p}<0.01$ ) during the current recession. As shown in the figure, those countries which have instituted greater increases in government spending have had larger rises in per capita GDP.

Second, to further test the generalizability of our findings, we performed an out-of-sample prediction using GDP data on the US and Japan. As shown in Figure 4, during the recessionary years of 2008-2010, both US

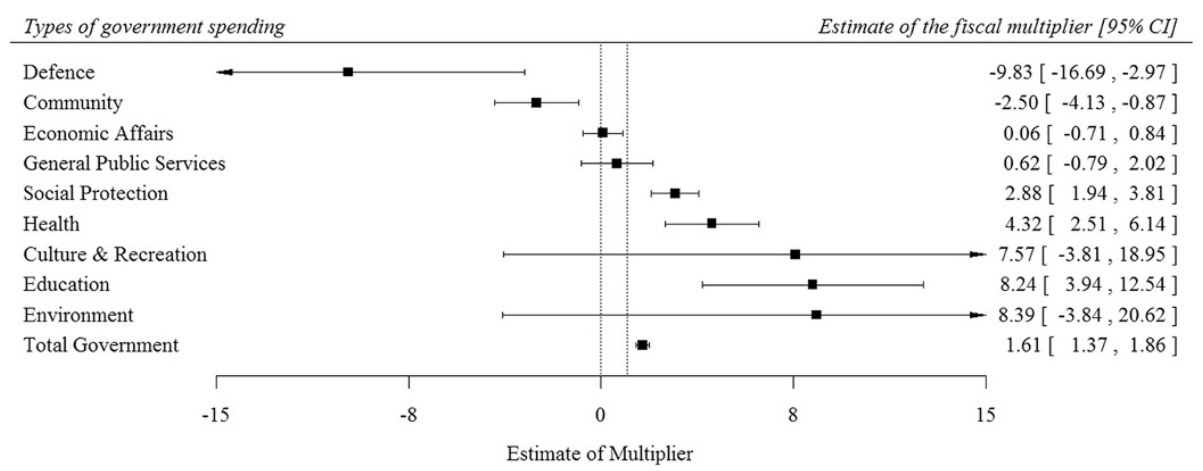

Figure 1 Pre-recession fiscal multipliers, 1995-2007. 


\section{a Pre-Recession Fiscal Multipliers 1995-2007, adjusted for time dummies}

Types of government spending

Estimate of the fiscal multiplier [95\% CI]

\begin{tabular}{|c|c|c|c|}
\hline Defence & $\longrightarrow$ & & $-7.28[-14.17,-0.39]$ \\
\hline Community & $\longmapsto$ & & $-1.32[-2.34,-0.29]$ \\
\hline Economic Affairs & & - & $0.87[0.18,1.55]$ \\
\hline General Public Services & & $=$ & $1.80[0.46,3.15]$ \\
\hline Social Protection & & 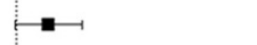 & $1.96[0.97,2.95]$ \\
\hline Health & & $\longrightarrow$ & $2.40[0.25,4.54]$ \\
\hline Environment & & & $3.98[-3.78,11.75]$ \\
\hline Culture \& Recreation & & - & $4.02[-2.80,10.83]$ \\
\hline Education & $\vdash$ & 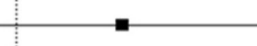 & $4.13[-0.77,9.03]$ \\
\hline Total Government & & - & $1.42[1.11,1.74]$ \\
\hline$\Gamma$ & 1 & $T$ & $\neg$ \\
\hline-15 & -8 & 8 & 15 \\
\hline
\end{tabular}

b Pre-Recession Fiscal Multipliers, 1995-2007, adjusted for interest rates, unemployment, trade balance, and domestic investment

Types of government spending

Estimate of the fiscal multiplier [95\% CI]

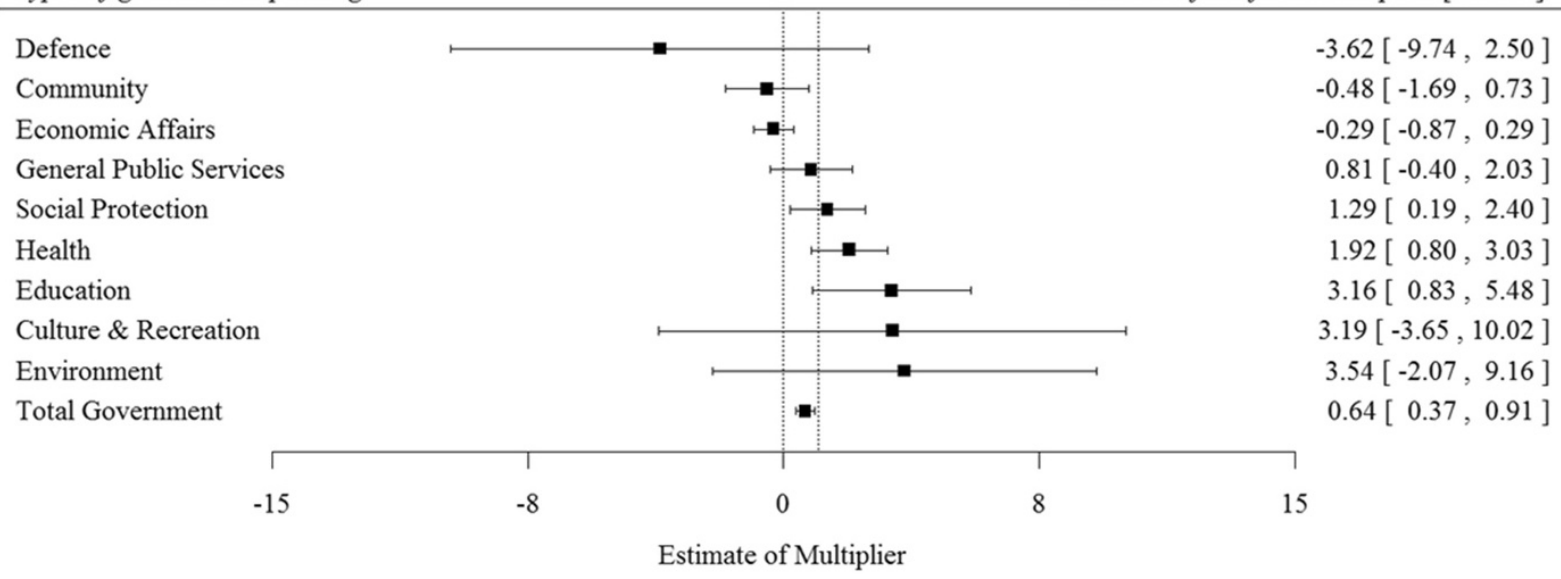

C Pre-Recession Fiscal Multipliers, 1995-2007, adjusted for interest rates, unemployment, trade balance, domestic investment and time dummies

Types of government spending

Estimate of the fiscal multiplier [95\% CI]

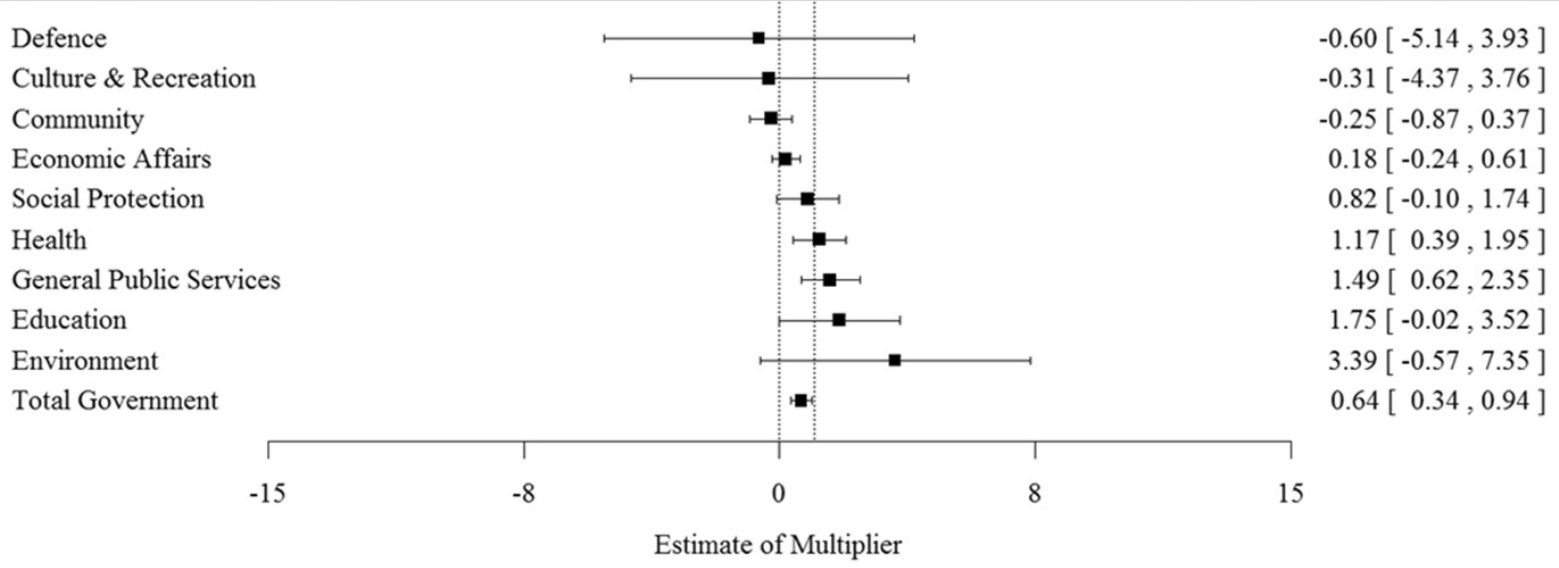

Figure 2 Adjusted pre-recession fiscal multipliers 1995-2007, by type of government spending. a. Pre-recession fiscal multipliers 1995-2007, adjusted for time dummies, 1995-2007. b. Pre-recession fiscal multipliers, 1995-2007, adjusted for interest rates, unemployment, trade balance, and domestic investment. c. Pre-recession fiscal multipliers, 1995-2007, adjusted for interest rates, unemployment, trade balance, domestic investment and time dummies. 
Table 2 Estimated fiscal multipliers, adjusted for unemployment rates, interest rates, trade balance (net exports), and domestic investment

a. Fiscal multiplier for total government spending

\begin{tabular}{|c|c|c|c|c|c|}
\hline \multirow[b]{2}{*}{ Covariates } & \multicolumn{5}{|c|}{ Gross domestic product adjusted for inflation and purchasing-power parity } \\
\hline & $(1)$ & $(2)$ & (3) & (4) & $(5)$ \\
\hline \multirow[t]{2}{*}{ Total government spending } & $1.61^{* *}$ & $1.60^{* *}$ & $1.38^{* *}$ & $1.10^{* *}$ & $0.64^{* *}$ \\
\hline & $(0.12)$ & $(0.15)$ & $(0.17)$ & $(0.10)$ & $(0.13)$ \\
\hline \multirow[t]{2}{*}{ Unemployment rate } & - & $-248.28^{* *}$ & $-258.67^{* *}$ & $-364.58^{* *}$ & -205.14 \\
\hline & & $(65.84)$ & $(86.17)$ & $(94.99)$ & $(131.78)$ \\
\hline \multirow[t]{2}{*}{ Interest rate } & - & - & $-320.26^{* *}$ & $-304.69^{* *}$ & $-269.42^{* *}$ \\
\hline & & & $(94.24)$ & $(76.57)$ & $(54.26)$ \\
\hline \multirow[t]{2}{*}{ Net exports (PPP, Real, per capita) } & - & - & - & $0.61^{* *}$ & $1.31^{* *}$ \\
\hline & & & & $(0.16)$ & $(0.19)$ \\
\hline \multirow[t]{2}{*}{ Total domestic investment (PPP, Real, per capita) } & - & - & - & - & $1.12^{* *}$ \\
\hline & & & & & $(0.26)$ \\
\hline Country-years & 296 & 213 & 213 & 213 & 192 \\
\hline Number of countries & 25 & 19 & 19 & 19 & 19 \\
\hline
\end{tabular}

\section{b. Fiscal multiplier for health spending}

Gross domestic product adjusted for inflation and purchasing-power parity

\begin{tabular}{|c|c|c|c|c|c|}
\hline Covariates & (1) & $(2)$ & (3) & (4) & (5) \\
\hline \multirow[t]{2}{*}{ Total health spending } & $4.32^{* *}$ & $3.21^{* *}$ & 2.07 & $2.23^{*}$ & $1.92^{* *}$ \\
\hline & $(0.88)$ & $(0.98)$ & $(1.21)$ & $(0.90)$ & $(0.53)$ \\
\hline \multirow[t]{2}{*}{ Total government spending minus health spending } & $1.05^{* *}$ & $1.26^{* *}$ & $1.25^{* *}$ & $0.88^{* *}$ & $0.39^{*}$ \\
\hline & $(0.33)$ & $(0.37)$ & $(0.39)$ & $(0.27)$ & $(0.15)$ \\
\hline \multirow[t]{2}{*}{ Unemployment rate } & - & $-205.61^{* *}$ & $-239.03^{*}$ & $-336.54^{* *}$ & -173.88 \\
\hline & & $(57.89)$ & $(87.57)$ & $(94.16)$ & $(123.10)$ \\
\hline \multirow[t]{2}{*}{ Interest rate } & - & - & $-286.44^{* *}$ & $-248.57^{* *}$ & $-203.53^{* *}$ \\
\hline & & & $(83.05)$ & (58.98) & $(47.45)$ \\
\hline \multirow[t]{2}{*}{ Net exports (PPP, Real, per capita) } & - & - & - & $0.64^{* *}$ & $1.24^{* *}$ \\
\hline & & & & $(0.17)$ & $(0.17)$ \\
\hline \multirow[t]{2}{*}{ Total domestic investment (PPP, Real, per capita) } & - & - & - & - & $1.06^{* *}$ \\
\hline & & & & & $(0.25)$ \\
\hline Country-years & 296 & 213 & 213 & 213 & 192 \\
\hline Number of countries & 25 & 19 & 19 & 19 & 19 \\
\hline \multicolumn{6}{|l|}{ c. Fiscal multiplier for defense spending } \\
\hline & \multicolumn{5}{|c|}{ Gross domestic product adjusted for inflation and purchasing-power parity } \\
\hline Covariates & $(1)$ & $(2)$ & (3) & (4) & (5) \\
\hline \multirow[t]{2}{*}{ Total defense spending } & $-9.83^{* *}$ & $-13.17^{* *}$ & $-12.92^{* *}$ & -8.51 & -3.62 \\
\hline & $(3.32)$ & $(3.67)$ & $(4.33)$ & $(4.77)$ & $(2.91)$ \\
\hline \multirow[t]{2}{*}{ Total government spending minus defense spending } & $1.66^{* *}$ & $1.62^{* *}$ & $1.42^{* *}$ & $1.20^{* *}$ & $0.71^{* *}$ \\
\hline & $(0.08)$ & $(0.09)$ & $(0.10)$ & $(0.11)$ & $(0.14)$ \\
\hline \multirow[t]{2}{*}{ Unemployment rate } & - & $-286.58^{* *}$ & $-301.73^{* *}$ & $-368.95^{* *}$ & -221.27 \\
\hline & & $(66.15)$ & $(90.73)$ & $(91.27)$ & $(124.46)$ \\
\hline \multirow[t]{2}{*}{ Interest rate } & - & - & $-253.31^{*}$ & $-263.02^{* *}$ & $-251.73^{* *}$ \\
\hline & & & $(96.90)$ & (84.62) & $(55.96)$ \\
\hline \multirow[t]{2}{*}{ Net exports (PPP, Real, per capita) } & - & - & - & $0.47^{*}$ & $1.24^{* *}$ \\
\hline & & & & $(0.20)$ & $(0.18)$ \\
\hline
\end{tabular}


Table 2 Estimated fiscal multipliers, adjusted for unemployment rates, interest rates, trade balance (net exports), and domestic investment (Continued)

\begin{tabular}{lcccc}
\hline Total domestic investment (PPP, Real, per capita) & - & - & - & $1.08^{* *}$ \\
& & & 213 & $(0.25)$ \\
Country-years & 296 & 213 & 19 & 192 \\
Number of countries & 25 & 19 & 19 & 19
\end{tabular}

\section{d. Fiscal multiplier for education spending}

Gross domestic product adjusted for inflation and purchasing-power parity

\begin{tabular}{|c|c|c|c|c|c|}
\hline Covariates & (1) & (2) & (3) & (4) & (5) \\
\hline \multirow[t]{2}{*}{ Total education spending } & $8.24^{* *}$ & $6.53^{* *}$ & 4.98 & $5.06^{* *}$ & $3.16^{*}$ \\
\hline & $(2.09)$ & $(2.10)$ & $(2.50)$ & $(1.75)$ & $(1.11)$ \\
\hline \multirow[t]{2}{*}{ Total government spending minus education spending } & $0.66^{*}$ & $0.88^{* *}$ & $0.92^{* *}$ & $0.59^{* *}$ & 0.33 \\
\hline & $(0.24)$ & $(0.26)$ & $(0.27)$ & $(0.20)$ & $(0.24)$ \\
\hline \multirow[t]{2}{*}{ Unemployment rate } & - & $-188.09^{* *}$ & $-227.19^{* *}$ & $-333.20^{* *}$ & -168.63 \\
\hline & & $(58.30)$ & $(76.03)$ & $(84.09)$ & $(117.61)$ \\
\hline \multirow[t]{2}{*}{ Interest rate } & - & - & -211.45 & -184.21 & $-186.19^{* *}$ \\
\hline & & & (127.38) & $(97.00)$ & $(61.05)$ \\
\hline \multirow[t]{2}{*}{ Net exports (PPP, Real, per capita) } & - & - & - & $0.63^{* *}$ & $1.26^{* *}$ \\
\hline & & & & $(0.15)$ & $(0.17)$ \\
\hline \multirow[t]{2}{*}{ Total domestic investment (PPP, Real, per capita) } & - & - & - & - & $1.11^{* *}$ \\
\hline & & & & & $(0.22)$ \\
\hline Country-years & 296 & 213 & 213 & 213 & 192 \\
\hline Number of countries & 25 & 19 & 19 & 19 & 19 \\
\hline
\end{tabular}

e. Fiscal multiplier for general public services spending

Gross domestic product adjusted for inflation and purchasing-power parity

\begin{tabular}{|c|c|c|c|c|c|}
\hline Covariates & $(1)$ & $(2)$ & (3) & (4) & $(5)$ \\
\hline \multirow[t]{2}{*}{ Total general public services spending } & 0.62 & 1.37 & $2.44^{*}$ & $2.05^{*}$ & 0.81 \\
\hline & $(0.68)$ & $(0.71)$ & $(0.89)$ & $(0.88)$ & $(0.58)$ \\
\hline \multirow{2}{*}{$\begin{array}{l}\text { Total government spending minus general public services } \\
\text { spending }\end{array}$} & $1.75^{* *}$ & $1.63^{* *}$ & $1.21^{* *}$ & $0.96^{* *}$ & $0.62^{* *}$ \\
\hline & $(0.09)$ & $(0.12)$ & $(0.14)$ & $(0.14)$ & $(0.11)$ \\
\hline \multirow[t]{2}{*}{ Unemployment rate } & - & $-242.48^{* *}$ & $-279.25^{* *}$ & $-381.55^{* *}$ & -207.43 \\
\hline & & $(67.58)$ & $(88.70)$ & $(100.42)$ & $(132.83)$ \\
\hline \multirow[t]{2}{*}{ Interest rate } & - & - & $-396.26^{* *}$ & $-372.92^{* *}$ & $-284.09^{* *}$ \\
\hline & & & $(97.04)$ & $(84.44)$ & $(59.63)$ \\
\hline \multirow[t]{2}{*}{ Net exports (PPP, Real, per capita) } & - & - & - & $0.60^{* *}$ & $1.30^{* *}$ \\
\hline & & & & $(0.16)$ & $(0.19)$ \\
\hline \multirow[t]{2}{*}{ Total domestic investment (PPP, Real, per capita) } & - & - & - & - & $1.12^{* *}$ \\
\hline & & & & & $(0.27)$ \\
\hline Country-years & 296 & 213 & 213 & 213 & 192 \\
\hline Number of countries & 25 & 19 & 19 & 19 & 19 \\
\hline \multicolumn{6}{|l|}{ f. Fiscal multiplier for culture \& recreation spending } \\
\hline & \multicolumn{5}{|c|}{ Gross domestic product adjusted for inflation and purchasing-power parity } \\
\hline Covariates & $(1)$ & (2) & $(3)$ & (4) & $(5)$ \\
\hline \multirow[t]{2}{*}{ Total culture \& recreation spending } & 7.57 & 6.25 & 4.52 & 0.83 & 3.19 \\
\hline & $(5.51)$ & $(4.70)$ & $(3.99)$ & $(2.96)$ & $(3.25)$ \\
\hline
\end{tabular}


Table 2 Estimated fiscal multipliers, adjusted for unemployment rates, interest rates, trade balance (net exports), and domestic investment (Continued)

\begin{tabular}{|c|c|c|c|c|c|}
\hline \multirow{2}{*}{$\begin{array}{l}\text { Total government spending minus culture \& recreation } \\
\text { spending }\end{array}$} & $1.34^{* *}$ & $1.38^{* *}$ & $1.24^{* *}$ & $1.11^{* *}$ & $0.57^{* *}$ \\
\hline & $(0.32)$ & $(0.30)$ & $(0.26)$ & $(0.19)$ & (0.18) \\
\hline \multirow{2}{*}{ Unemployment rate } & - & $-243.28^{* *}$ & $-259.08^{* *}$ & $-365.32^{* *}$ & -194.27 \\
\hline & & $(65.52)$ & (88.99) & $(94.63)$ & $(129.50)$ \\
\hline \multirow[t]{2}{*}{ Interest rate } & - & - & $-306.23^{* *}$ & $-305.79 * *$ & $-258.23^{* *}$ \\
\hline & & & $(98.71)$ & $(77.59)$ & $(54.23)$ \\
\hline \multirow[t]{2}{*}{ Net exports (PPP, Real, per capita) } & - & - & - & $0.61^{* *}$ & $1.31^{* *}$ \\
\hline & & & & (0.16) & $(0.18)$ \\
\hline \multirow[t]{2}{*}{ Total domestic investment (PPP, Real, per capita) } & - & - & - & - & $1.14^{* *}$ \\
\hline & & & & & $(0.26)$ \\
\hline Country-years & 296 & 213 & 213 & 213 & 192 \\
\hline Number of countries & 25 & 19 & 19 & 19 & 19 \\
\hline
\end{tabular}

g. Fiscal multiplier for community spending

Gross domestic product adjusted for inflation and purchasing-power parity

\begin{tabular}{|c|c|c|c|c|c|}
\hline Covariates & $(1)$ & $(2)$ & $(3)$ & (4) & (5) \\
\hline \multirow[t]{2}{*}{ Total community spending } & $-2.50^{* *}$ & $-2.32^{* *}$ & $-1.57^{* *}$ & -0.91 & -0.48 \\
\hline & $(0.79)$ & $(0.63)$ & $(0.52)$ & $(0.63)$ & (0.58) \\
\hline \multirow[t]{2}{*}{ Total government spending minus community spending } & $1.68^{* *}$ & $1.67^{* *}$ & $1.47^{* *}$ & $1.19^{* *}$ & $0.73^{* *}$ \\
\hline & $(0.10)$ & $(0.13)$ & $(0.15)$ & $(0.09)$ & $(0.11)$ \\
\hline \multirow[t]{2}{*}{ Unemployment rate } & - & $-231.99^{* *}$ & $-255.56^{* *}$ & $-351.38^{* *}$ & -198.19 \\
\hline & & $(58.31)$ & $(85.00)$ & $(90.87)$ & $(129.65)$ \\
\hline \multirow[t]{2}{*}{ Interest rate } & - & - & $-254.50^{* *}$ & $-260.82^{* *}$ & $-248.63^{* *}$ \\
\hline & & & $(80.86)$ & $(69.72)$ & $(49.31)$ \\
\hline \multirow[t]{2}{*}{ Net exports (PPP, Real, per capita) } & - & - & - & $0.55^{* *}$ & $1.22^{* *}$ \\
\hline & & & & $(0.14)$ & $(0.19)$ \\
\hline \multirow[t]{2}{*}{ Total domestic investment (PPP, Real, per capita) } & - & - & - & - & $1.03^{* *}$ \\
\hline & & & & & $(0.28)$ \\
\hline Country-years & 296 & 213 & 213 & 213 & 192 \\
\hline Number of countries & 25 & 19 & 19 & 19 & 19 \\
\hline \multicolumn{6}{|l|}{ h. Fiscal multiplier for environment spending } \\
\hline & \multicolumn{5}{|c|}{ Gross domestic product adjusted for inflation and purchasing-power parity } \\
\hline Covariates & $(1)$ & $(2)$ & (3) & (4) & $(5)$ \\
\hline \multirow[t]{2}{*}{ Total environment spending } & 8.39 & 1.03 & -0.46 & 3.02 & 3.54 \\
\hline & $(5.92)$ & $(4.73)$ & $(4.01)$ & $(4.02)$ & $(2.67)$ \\
\hline \multirow[t]{2}{*}{ Total government spending minus environment spending } & $1.50^{* *}$ & $1.60^{* *}$ & $1.40^{* *}$ & $1.07^{* *}$ & $0.59^{* *}$ \\
\hline & $(0.17)$ & $(0.16)$ & (0.18) & $(0.14)$ & $(0.14)$ \\
\hline \multirow[t]{2}{*}{ Unemployment rate } & - & $-251.28^{* *}$ & $-269.12^{* *}$ & $-356.05^{* *}$ & -193.58 \\
\hline & & $(67.63)$ & $(86.71)$ & $(96.24)$ & $(128.60)$ \\
\hline \multirow[t]{2}{*}{ Interest rate } & - & - & $-322.65^{* *}$ & $-301.83^{* *}$ & $-261.57^{* *}$ \\
\hline & & & $(94.54)$ & $(78.69)$ & $(56.92)$ \\
\hline \multirow[t]{2}{*}{ Net exports (PPP, Real, per capita) } & - & - & - & $0.62^{* *}$ & $1.35^{* *}$ \\
\hline & & & & $(0.16)$ & $(0.18)$ \\
\hline \multirow[t]{2}{*}{ Total domestic investment (PPP, Real, per capita) } & - & - & - & - & $1.14^{* *}$ \\
\hline & & & & & $(0.26)$ \\
\hline
\end{tabular}


Table 2 Estimated fiscal multipliers, adjusted for unemployment rates, interest rates, trade balance (net exports), and domestic investment (Continued)

\begin{tabular}{|c|c|c|c|c|c|}
\hline Country-years & 296 & 213 & 213 & 213 & 192 \\
\hline Number of countries & 25 & 19 & 19 & 19 & 19 \\
\hline \multicolumn{6}{|l|}{ i. Fiscal multiplier for economic affairs spending } \\
\hline & \multicolumn{5}{|c|}{ Gross domestic product adjusted for inflation and purchasing-power parity } \\
\hline Covariates & (1) & (2) & (3) & (4) & (5) \\
\hline \multirow[t]{2}{*}{ Total economic affairs spending } & 0.06 & -0.06 & 0.03 & -0.55 & -0.29 \\
\hline & $(0.38)$ & $(0.45)$ & $(0.40)$ & $(0.35)$ & $(0.28)$ \\
\hline \multirow{2}{*}{$\begin{array}{l}\text { Total government spending minus economic affairs } \\
\text { spending }\end{array}$} & $1.83^{* *}$ & $1.80^{* *}$ & $1.58^{* *}$ & $1.32^{* *}$ & $0.84^{* *}$ \\
\hline & $(0.14)$ & $(0.17)$ & $(0.20)$ & $(0.14)$ & $(0.19)$ \\
\hline \multirow[t]{2}{*}{ Unemployment rate } & - & $-248.83^{* *}$ & $-271.11^{* *}$ & $-388.53^{* *}$ & -233.36 \\
\hline & & $(62.40)$ & $(75.94)$ & $(84.40)$ & (125.08) \\
\hline \multirow[t]{2}{*}{ Interest rate } & - & - & $-265.45^{* *}$ & $-237.34^{* *}$ & $-238.72^{* *}$ \\
\hline & & & $(91.29)$ & $(74.14)$ & $(50.65)$ \\
\hline \multirow[t]{2}{*}{ Net exports (PPP, Real, per capita) } & - & - & - & $0.66^{* *}$ & $1.27^{* *}$ \\
\hline & & & & $(0.13)$ & $(0.18)$ \\
\hline \multirow[t]{2}{*}{ Total domestic investment (PPP, Real, per capita) } & - & - & - & - & $0.97^{* *}$ \\
\hline & & & & & $(0.26)$ \\
\hline Country-years & 296 & 213 & 213 & 213 & 192 \\
\hline Number of countries & 25 & 19 & 19 & 19 & 19 \\
\hline
\end{tabular}

j. Fiscal multiplier for social protection spending

Gross domestic product adjusted for inflation and purchasing-power parity

\begin{tabular}{|c|c|c|c|c|c|}
\hline Covariates & (1) & (2) & (3) & (4) & (5) \\
\hline \multirow[t]{2}{*}{ Total social protection spending } & $2.88^{* *}$ & $3.25^{* *}$ & $2.72^{* *}$ & $2.21^{* *}$ & $1.29^{*}$ \\
\hline & $(0.45)$ & $(0.49)$ & $(0.64)$ & $(0.49)$ & $(0.53)$ \\
\hline \multirow{2}{*}{$\begin{array}{l}\text { Total government spending minus social protection } \\
\text { spending }\end{array}$} & $0.83^{* *}$ & 0.52 & 0.57 & $0.47^{*}$ & 0.36 \\
\hline & $(0.27)$ & $(0.26)$ & $(0.31)$ & $(0.20)$ & $(0.20)$ \\
\hline \multirow[t]{2}{*}{ Unemployment rate } & - & $-289.73^{* *}$ & $-323.90^{* *}$ & $-410.46^{* *}$ & -254.49 \\
\hline & & $(61.01)$ & (85.98) & (94.19) & $(142.36)$ \\
\hline \multirow[t]{2}{*}{ Interest rate } & - & - & -211.82 & $-217.75^{*}$ & $-229.26^{* *}$ \\
\hline & & & $(101.48)$ & $(80.13)$ & $(59.17)$ \\
\hline \multirow[t]{2}{*}{ Net exports (PPP, Real, per capita) } & - & - & - & $0.57^{* *}$ & $1.28^{* *}$ \\
\hline & & & & $(0.17)$ & $(0.21)$ \\
\hline \multirow[t]{2}{*}{ Total domestic investment (PPP, Real, per capita) } & - & - & - & - & $0.98^{* *}$ \\
\hline & & & & & $(0.31)$ \\
\hline Country-years & 296 & 213 & 213 & 213 & 192 \\
\hline Number of countries & 25 & 19 & 19 & 19 & 19 \\
\hline
\end{tabular}

Notes: Robust standard errors in parentheses clustered by country to reflect non-independence of sampling.

** $p<0.01,{ }^{*} p<0.05$.

and Japan's economic performance were predicted by changes in total government spending.

\section{Comparing recession and pre-recession multipliers}

To test whether fiscal multipliers differed across recessionary and non-recessionary periods, we extended our disaggregated analysis of fiscal multipliers of 1995-2007 to include 2008-2010. As shown in Table 3, there was a significant interaction of the recessionary period with the estimated magnitude of fiscal multipliers. Apart from defence and public service spending, the fiscal multipliers were slightly attenuated during the recessionary period. The multiplier for total government spending, for example, was reduced by 0.15 (95\% CI: 0.08 to 0.21 ). 


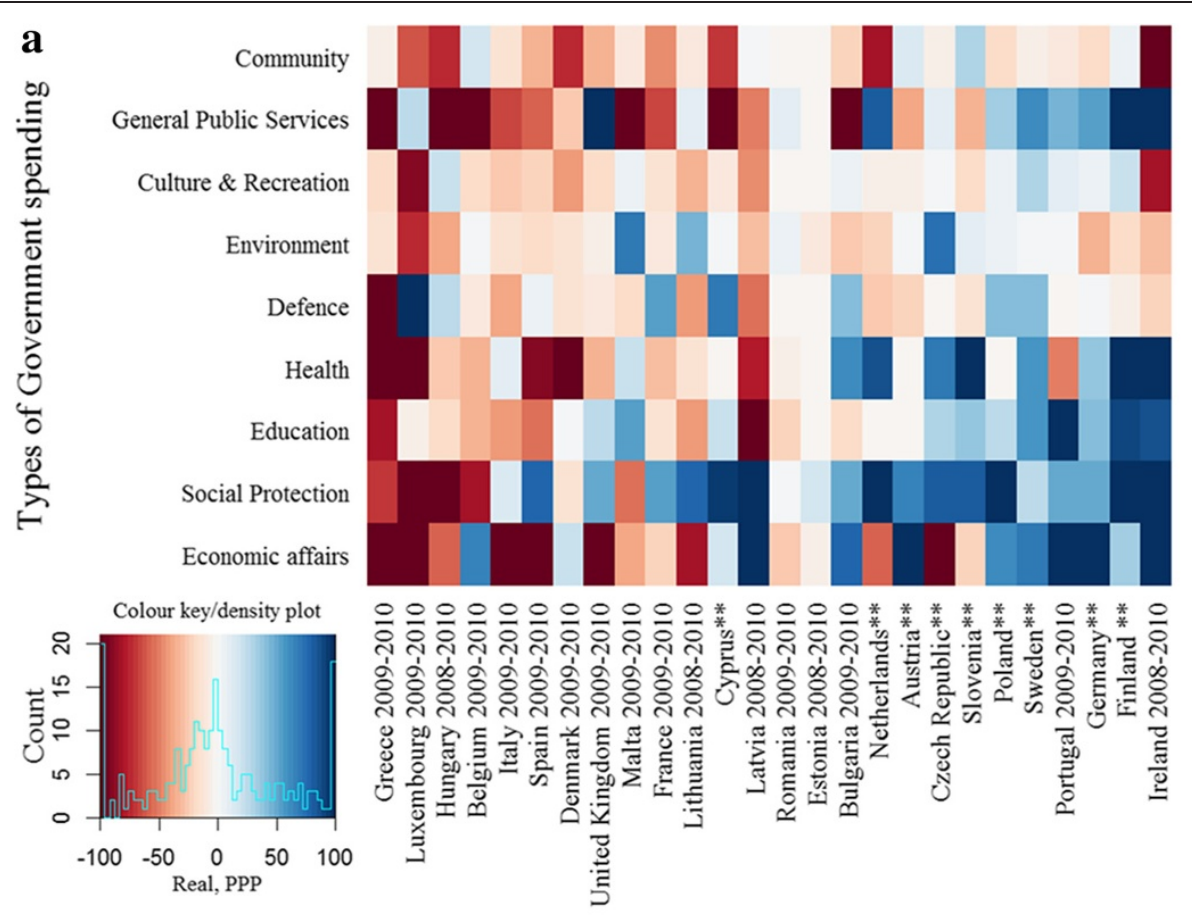

b

\section{Country}

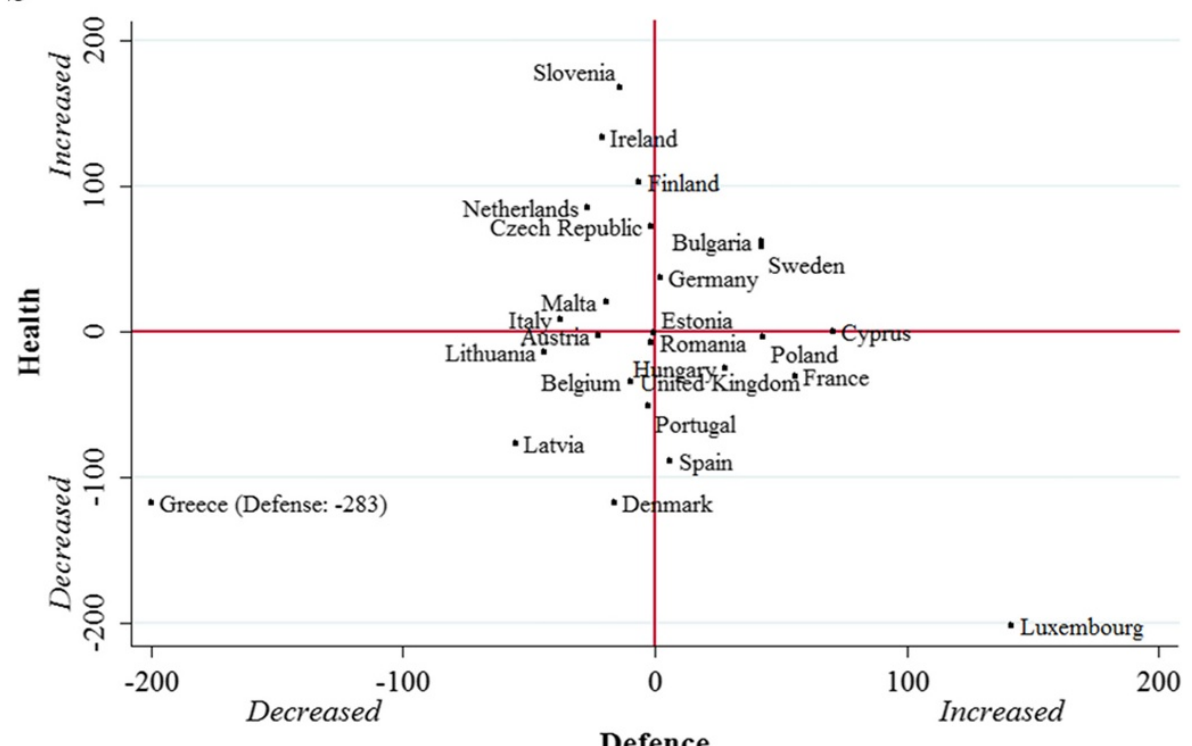

Defence Note: Greece ( $x$-axis) and Iceland ( $y$-axis) have been rescaled to bring them within the
range of the plot. The data in the parentheses shows the actual result for the scaled axis

Correlation Coefficient: $-0.04(p=0.85)$

Figure 3 Budgetary patterns and effects. Panel a. Heat map of budget changes in Europe. Panel b. Association of health with defence spending.

Health and education dropped, respectively, by 1.31 (95\%: 0.94 to 1.68 ) and 1.44 (95\% CI: 0.80 to 2.08). Social protection spending was reduced to a lesser degree, by 0.33 (95\%: 0.11 to 0.56). However, in the statistical models, the health, education, and social protection categories of spending all exhibited statistically significant and positive growth associations above 1 during the recessionary period.

\section{Sensitivity analysis}

We tested the robustness of multiplier estimates to several alternative model specifications. First, we incorporated a 


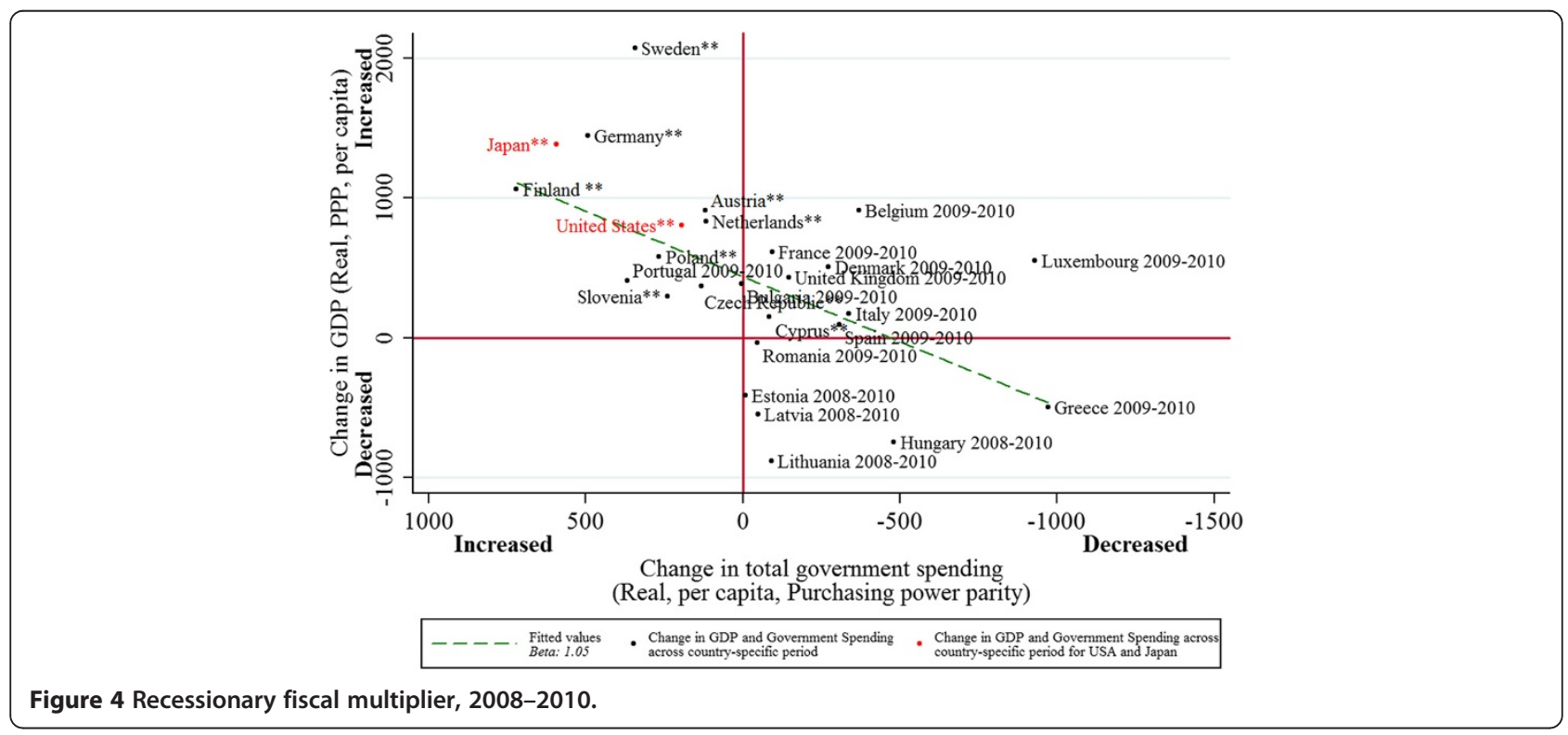

set of time dummies to correct for cross-EU economic interactions, finding that none of the results was qualitatively changed (see Figure $2 \mathrm{~b}$ ). Second, we included both time dummies and the aforementioned set of controls and found that direction of the coefficients remained the same and the health multiplier remained above 1 in the prerecessionary period (see Figure 2c). Third, we used alternative data sources of government statistics, including the OECD and World Bank. None of the spending multipliers qualitatively changed.

To test whether budgetary changes in the recessionary period were confounded by the depth of recession (peak-to-trough change in GDP), we assessed the correlation of depth of recession and the depth of subsequent government spending changes $(r=0.12, \mathrm{p}=0.59)$. None of the results was changed when controlling for the cumulative magnitude of recession. Overall, we observed no correlation among changes in government spending instituted in different sectors (e.g., changes in health and defence spending are not correlated, $r=0.02, p=0.90$, panel $b$ of Figure 3), indicating large, exogenous variation in budgetary choices in response to recession.

\section{Discussion}

Government spending changes during recession serves as a large "quasi-natural experiment", in which countries experiencing relatively similar recessions undertook alternative budgetary paths. From these differences, we can deduce the relationship between spending and economic outcomes. Our findings suggest a total pro-growth effect of overall government spending, with significant positive fiscal multipliers in the social protection, health and education sectors [29]. These estimates were based on crossnational models addressing potential economic and political confounding factors. By empirically evaluating fiscal multipliers across different sectors, we observed that changes in government spending corresponded to similar directional changes in economic growth during non-recessionary and recessionary periods. Multipliers estimated from non-recession periods can account for current changes in economic growth associated with spending instituted in 2008-2010.

Our study has several limitations. First, the statistical analysis covers only the first three years of economic recession, given the limited availability of more recent data. While it has been argued that short-term multipliers may be fail to account for longer-term effects [30], we found that estimated fiscal multipliers from 19952007 predicted directional changes in subsequent shortterm periods. However, it is possible that longer-term effects differ from those observed currently, remaining an important topic for future research. Second, there was marked heterogeneity in the magnitude of fiscal multipliers. This heterogeneity exists both within and between countries. Within countries, our results identified that domestic absorption of spending is a significant factor mediating the relationship between government spending and economic growth. When we adjusted for the trade balance, the multipliers for components of government spending which are tradable, such as defence, were attenuated, whereas semi-tradable components, such as health and education, were not significantly altered. Across countries, the magnitude of the fiscal multiplier is also likely to depend on several country-specific factors and market characteristics, including the level of economic development, exchange rate regime, openness to trade, and public debt dynamics [31,32]. Third, the data on government spending could not differentiate the 
Table 3 Recessionary (1995-2010) and pre-recession fiscal multipliers (1995-2007), by type of government spending

\begin{tabular}{|c|c|c|c|}
\hline \multirow[b]{2}{*}{ Covariates } & \multicolumn{3}{|c|}{ Increase in GDP (PPP, per capita, real) } \\
\hline & $\begin{array}{l}\text { Estimated } \\
\text { multiplier } \\
1995-2010\end{array}$ & $\begin{array}{l}\text { Recession } \\
\text { interaction }\end{array}$ & $\begin{array}{l}\text { Estimated } \\
\text { recession } \\
\text { multiplier }\end{array}$ \\
\hline & (1) & (2) & (3) \\
\hline \multirow{2}{*}{$\begin{array}{l}\text { Total Government } \\
\text { Spending }\end{array}$} & 1.28 & -0.15 & 1.13 \\
\hline & (0.78 to 1.77$)$ & $(-0.084$ to -0.21$)$ & (0.66 to 1.61$)$ \\
\hline \multirow[t]{2}{*}{ Defence } & -5.69 & -1.71 & -7.40 \\
\hline & $(-15.77$ to 4.39$)$ & $(-6.16$ to 2.74$)$ & $(-18.12$ to 3.32$)$ \\
\hline \multirow[t]{2}{*}{ Community } & -2.29 & -5.99 & -8.29 \\
\hline & $(-4.84$ to 0.25$)$ & $(-1.34$ to -10.64$)$ & $(-13.45$ to -3.12$)$ \\
\hline \multirow[t]{2}{*}{ Economic Affairs } & 0.45 & -1.17 & -0.72 \\
\hline & $(-0.35$ to 1.24$)$ & $(-1.99$ to -0.35$)$ & $(-1.09$ to -0.35$)$ \\
\hline \multirow{2}{*}{$\begin{array}{l}\text { General Public } \\
\text { Services }\end{array}$} & 1.57 & -0.45 & 1.12 \\
\hline & $(-0.26$ to 3.40$)$ & $(-1.17$ to 0.28$)$ & $(-0.75$ to 3.00$)$ \\
\hline \multirow[t]{2}{*}{ Social Protection } & 3.04 & -0.33 & 2.71 \\
\hline & (2.05 to 4.03 ) & $(-0.56$ to -0.11$)$ & (1.81 to 3.60$)$ \\
\hline \multirow[t]{2}{*}{ Health } & 4.92 & -1.31 & 3.61 \\
\hline & (2.92 to 6.93 ) & $(-1.68$ to -0.94$)$ & (1.60 to 5.62$)$ \\
\hline \multirow{2}{*}{$\begin{array}{l}\text { Culture \& } \\
\text { Recreation }\end{array}$} & 14.12 & -2.38 & 11.83 \\
\hline & (2.16 to 26.26$)$ & $(-0.67$ to -4.10$)$ & $(-0.92$ to 24.58$)$ \\
\hline \multirow[t]{2}{*}{ Education } & 9.37 & -1.44 & 7.92 \\
\hline & (4.40 to 14.34 ) & $(-2.08$ to -0.80$)$ & (3.19 to 12.66 ) \\
\hline \multirow[t]{2}{*}{ Environment } & 9.49 & -6.30 & 3.18 \\
\hline & $(-5.03$ to 24.00$)$ & $(-8.93$ to -3.68$)$ & $(-10.60$ to 16.97$)$ \\
\hline
\end{tabular}

Notes: Numbers in parentheses are $95 \%$ confidence intervals. GDP and all forms of government spending are adjusted for inflation and purchasing-power parity. Robust standard errors in parentheses clustered by country to reflect non-independence of sampling Columns 1-2 report coefficients from equation 2. Column 3 reports the linear combination of columns 1 and 2. All models control for between-country variation.

growth effects of government spending that acts as a stimulus from those effects of spending which replaces spending during recessions. Although it has been suggested that recessionary periods exhibit higher multiplier effects, as consumer demand is relatively low, we found contrary evidence that fiscal multipliers were slightly attenuated during periods of recessions compared with periods of economic growth. This may reflect a tendency of 'replacement' spending, such as when patients turn from private health care to public health care consumption during recessions, to have smaller pro-growth effects than 'stimulus' spending, which creates new jobs and income. This interpretation is corroborated by the observation that health spending was more greatly attenuated than total social protection, as the latter is largely driven by replacement spending both in recessionary and non-recessionary periods. Another possibility is that when multiple countries simultaneously introduce budget reductions, as in the current recession, the potential multiplier effects of government spending are dampened. Further disaggregating categories of government spending, identifying time lags, and evaluating the role of effect modifiers associated with various forms of government spending would be a next logical step for research to identify critical policy strategies during and after the ongoing economic recession.

Overall, our findings are consistent with recent estimates of fiscal multipliers for total government spending [28], but extends these estimates in a novel way to evaluate alternative budgetary sectors, compare periods of non-recession and recession, identify the role of domestic absorption, and include both Europe and the United States. It also lends support for theories that there are both 'productive' and 'unproductive' components of government spending [33]. However, in contrast with previous casestudy estimates of the fiscal multipliers associated with defence spending in the United States $[3,18,19]$, we estimated negative fiscal multipliers for military spending in Europe. This observation may reflect the presence of a large defense manufacturing sector in the United States so that most expenditure is absorbed domestically while, in most European countries, equipment is imported. It is also plausible that military spending will vary according to where the funds are being spent, so the multiplier effect may be greater in countries with a large domestic defence industry.

These findings have important implications for policy. First, these results, taken together with other studies, corroborates existing evidence that historical prescriptions for austerity from international financial institutions have tended to exacerbate economic crises $[29,34,35]$. Second, there is a widespread consensus that investment in education and in health contribute to economic growth in the long term, by creating a healthier, better educated, and therefore more productive labour force [36]. However, that argument finds little favour among those who view short term reductions in expenditure as a necessary condition for the recovery that will permit such investment in the future. Our findings suggest that, in addition to their long-term benefits, such investments may actually have short-term, positive growth effects that make that recovery more likely.

\section{Additional file}

Additional file 1: Web Appendix 1. Associations between trade balance and sector-specific government spending between 1995-2007, 26 EU countries.

\section{Competing interests}

We declare that we have no conflicts of interests. 


\section{Authors' contributions}

AR DS analyzed the data and wrote the draft; SB MM CM assisted with the analysis and helped finalise the paper. All authors read and approved the final manuscript.

\section{Funding}

AR and DS are supported by an Demetriq EU FP7 grant. This study was carried out with financial support from the Commission of the European Communities, Grant Agreement no. 278511. The study does not necessarily reflect the Commission's views and in no way anticipates the Commission's future policy in this area.

\section{Author details}

'Department of Sociology, University of Oxford, Manor Road Building, Manor Road, Oxford OX1 3UQ, England. 'Stanford Prevention Research Center, Department of Medicine, Stanford University, Palo Alto, CA, USA. ${ }^{3}$ London School of Hygiene \& Tropical Medicine, Department of Public Health and Policy, 15-17 Tavistock Place, London WC1H 9SH, UK. ${ }^{4}$ Department of Economics, University of California Davis, Davis, USA.

Received: 14 June 2013 Accepted: 20 August 2013 Published: 23 September 2013

\section{References}

1. Gali J, Lopez-Salido J, Valles J: Understanding the effects of government spending on consumption. J Eur Econ Assoc 2010, 5:227-270.

2. Brown C: Fiscal policy in the thirties: a reappraisal. Am Econ Rev 1956, 46:857-859.

3. Romer C, Bernstein J: The job impact of the American recovery and reinvestment plan, Report from the Council of Economic Advisers, January. 2009

4. Giavazzi F, Pagano M: Can severe fiscal contractions be expansionary? Tales of two small European countries. NBER Working Paper No 3372. Cambridge, MA: MIT Press; 1990.

5. Taylor J: Macroeconomic policy in a world economy: from econometric design to practical operation. New York: WW Norton; 1993.

6. Correia-Nunes J, Stemitsiotis L: Budget deficit and interest rates: is there a link? International evidence. Oxford Bull Econ Stat 1995, 57:425-449.

7. Laubach T: New evidence on the interest rate effects of budget deficits and debt. J Eur Econ Assoc 2009, 7:858-885.

8. A B: How the Austerity Class Rules Washington, The Nation. 2011. http://www. thenation.com/article/164073/how-austerity-class-rules-washington\#.

9. Mundell RA: The appropriate use of monetary and fiscal policy for internal and external stability. Staff Papers - International Monetary Fund 1962, 9:70-79.

10. Evans P: Do budget deficits raise nominal interest rates?: Evidence from six countries. J Monetary Econ 1987, 20:281-300.

11. Evans P: Interest rates and expected future budget deficits in the United States. J Pol Econ 1987, 95:34-58.

12. Hoelscher GP: Federal borrowing and short term interest rates. South Econ J 1983, 50:319-333.

13. Aschaeur D: Does public capital crowd out private capital? J Monetary Econ 1989, 24:171-188.

14. Canning D: Infrastructure's Contribution to Aggregate Output, Policy Research Working Paper, no. 2246. Washington DC: World Bank; 1999.

15. Spilimbergo A, Symansky S, Blanchard O, Cottarelli C: Fiscal Policy for the Crisis, Book Fiscal Policy for the Crisis. IMF: City; 2008.

16. Spilimbergo A, Symansky S, Schindler M: Fiscal Multipliers, IMF Staff Positions, SPN/09/11. Washington, D.C: IMF; 2009.

17. International Monetary Fund: World Economic Outlook: Coping with High Debt and Sluggish Growth. Washington, D.C.: IMF; 2012.

18. Ramey VA: Identifying Government Spending Shocks: It's all in the Timing. The Quarterly Journal of Economics 2011, 126(1):1-50.

19. Ramey VA: Defense News Shocks, 1939-2008: An Analysis Based on News Sources, UCSD Working Paper. San Diego, CA: UCSD; 2009.

20. Blanchard O, Leigh D: Growth Forecast Errors and Fiscal Multipliers. Washington DC: IMF; 2013.

21. Scott A, Barnett S, De Broeck M, Ivanova A, Kim D, Kumhof M, Laxton D, Leigh D, Stehn SJ, Symansky S: Fiscal Policy as Countercyclical Tool. In World Economic Outlook: Financial Stress, Downturns, and Recoveries. Edited by Blanchard O. Washington, D.C., USA: IMF; 2008.
22. McKee M, Karanikolos M, Belcher P, Stuckler D: Austerity: a failed experiment on the people of Europe. Clinical Medicine 2012, 12:346-350.

23. Stuckler D, Basu S, Mckee M: Equitable distribution of pepfar-supported HIV/Aids services in South Africa respond. Am J Public Health 2011, 101:1351-1351.

24. EUROSTAT: General government expenditure by function. Luxembourg City, Luxembourg: EUROSTAT; 2012.

25. Auerbach AJ: The fall and rise of keynesian fiscal policy. Asian Econ Policy Rev 2012, 7:157-175.

26. Auerbach AJ, Gorodnichenko Y: Measuring the output responses to fiscal policy. Am Econ J: Econ Pol 2012, 4:1-27.

27. Stock JH, Watson MW: Vector autoregressions. J Econ Perspect 2001, 15:101-115.

28. Blanchard O, Perotti R: An empirical characterization of the dynamic effects of changes in government spending and taxes on output. Q J Econ 2002, 117:1329-1368.

29. Stuckler D, Basu S: The Body Economic: why austerity kills? London: Penguin; 2013.

30. Uhlig H: "Some Fiscal Calculus." American Economic Review 2010, 100(2):30-34.

31. IIzetzki E, Mendoza EG, Végh CA: How big (small?) are fiscal multipliers? J Monetary Econ 2012, 60(2):239-254.

32. Favero C, Giavazzi F, Perego J: Country Heterogeneity and the International: Evidence on the Effects of Fiscal Policy. NBER Working Paper 17272. Cambridge, MA: MIT Press; 2011.

33. Devarajan S, Swaroop V, Zou H: The composition of public expenditure and economic growth. Am Econ Rev 1996, 37:313-344.

34. Blyth M: Austerity: the history of a dangerous idea. Oxford: OUP; 2013.

35. Knight EKS: Analysis: Aid recipients welcome IMF's shift on austerity, Book Analysis: Aid recipients welcome IMFs shift on austerity. City; 2012. http://uk.reuters.com/ article/2012/10/14/us-imf-aid-admission-idUSBRE89D0GQ20121014.

36. Suhrcke M, McKee M, Stuckler D, Sauto Arce R, Tsolova S, Mortensen J: The contribution of health to the economy in the European Union. Public health 2006, 120:994-1001.

doi:10.1186/1744-8603-9-43

Cite this article as: Reeves et al:: Does investment in the health sector promote or inhibit economic growth? Globalization and Health 2013 9:43.

\section{Submit your next manuscript to BioMed Central and take full advantage of:}

- Convenient online submission

- Thorough peer review

- No space constraints or color figure charges

- Immediate publication on acceptance

- Inclusion in PubMed, CAS, Scopus and Google Scholar

- Research which is freely available for redistribution

Submit your manuscript at www.biomedcentral.com/submit
C Biomed Central 